\title{
Melan-A VLP Vaccine
}

National Cancer Institute

\section{Source}

National Cancer Institute. Melan-A VLP Vaccine. NCI Thesaurus. Code C61495.

A vaccine consisting of the melanocyte differentiation antigen Melan A (also called MART -1) encapsulated in noninfectious virus-like particles (VLP) with potential immunostimulating and antineoplastic activities. Upon administration, Melan-A VLP vaccine may activate the immune system to exert a specific cytotoxic T lymphocyte $(\mathrm{CTL})$ response against cancer cells expressing the Melan A antigen, resulting in tumor cell lysis. Melan A is an antigen that is upregulated in most melanomas. VLP stimulates the immune system and promotes the CT L response. 